\title{
ROLE OF GAS, SAMPLE AND LASER BEAM PARAMETERS IN LASER GENERATION OF A LOW THRESHOLD BREAKDOWN PLASMA IN A GAS IN FRONT OF A SOLID TARGET
}

\author{
J. HERMANN, C. BOULMER-LEBORGNE, I.N. MIHAILESCU* and B. DUBREUIL \\ G.R.E.M.I., Université d'Orléans, BP. 6759, F-45067 Orléans cedex 2, France \\ -Laser Department, Institute of Atomic Physics, Bucharest, Romania
}

\begin{abstract}
We studied the ignition process of the plasma which is created when a pulsed $\mathrm{CO}_{2}$ laser beam irradiates a (TI) metal sample in presence of an ambient gas. It is pointed out that the first step is due to target surface micro-defect vaporization. Then thermal ionization of the metal vapour occurs producing electrons which are heated by the inverse bremsstrahlung process. Finally, we observe an erosion plasma (which is dominated by the metal vapour) or a gas breakdown plasma, depending on the laser intensity and on the ambient gas properties. This can be described by the energy balance of plasma electrons and by the evaluation of material thermal heating.

Introduction

It is well-established nowadays that the optical breakdown of a particular gas can be obtained for much lower laser intensities $\left(10^{2}-10^{3}\right.$ for the IR-range) in front of a solid sample than in the free gas. The effect, which is called the "low-threshold optical gas breakdown", was demonstrated over the past years with all the known types of lasers emitting from the UV to the millimetric spectral range. Besides the scientific interest, the phenomenon was shown to have an important practical interest in designing new, more efficient laser processing technologies of various materials by taking advantage of the combined thermal, mechanical and chemical actions these plasmas excert upon solid surfaces.
\end{abstract}

Experimental part

In order to get a better insight into the features of plasma formation and evolution, special emphasis was put on the determination of the roles played in the incident laser radiation - plasma/gas - sample interaction processes by: (1) nature and pressure of the ambient gas, (11) the temporal shape of the laser pulses. Multipulse laser irradiation was performed with the ald of a TEA $\mathrm{CO} 2$ laser source. We cholced a metal target of titanium while the buffer gas was either $\mathrm{He}$, Ar or $\mathrm{N}_{2}$ at a pressure which was varied from a few Torr to atmospheric pressure. Plasma ignition and evolution were investigated by timeand space- resolved LIF- and emission- spectroscopy. The sample surface status was examined prior and after multipulse laser irradiation by electron microscopy.

Results

Theoretical evaluations show the existence of two thresholds upon laser intensity which must be achleved in order to get breakdown in the amblent gas. The first is the vaporization threshold (Iv), the second one is the gas breakdown ignition threshold (II). 
1) Vaporization threshold

A thermal analysis gives the temperature variation of the irradiated sample surface. Thus the laser light is considered to operate on the first layer containing impurities and micro-defects and presenting a specific absorptivity, (larger than pure metal one). The absorbed energy in this layer is evacuated into the bulk by thermal conduction. The target surface temperature varlation is obtained from the one dimensional heat conduction equation adjusted to the temporal shape of the laser pulse. Figs 1 and 2 show three pulse shapes and the corresponding surface temperature variations.

2) Breakdown threshold

The relatively high ionization degree $\left(10^{-5}-10^{-4}\right)$ of the metal vapour favours the inverse bremsstrahlung process. Calculations based on the gain-loss electron energy balance furnish a simple criterium for the gas breakdown plasma ignition:

$$
I_{1}\left[\mathrm{MW} \cdot \mathrm{cm}^{-2}\right]=C_{s t e} \frac{\Delta[\mathrm{eV}]}{\lambda^{2}\left[\mathrm{~m}^{2}\right] \mathrm{Mo}[\mathrm{amu}]}
$$

where $\Delta$ and Mo are lonization potential and atomic mass of the amblent gas or vapour, $\lambda$ is the laser wavelength and $I_{1}$ is the laser intensity threshold corresponding to the chosen temporal pulse shape.

In the case of $\mathrm{Ar}$ and $\mathrm{N}_{2}$ gases, we obtain $\mathrm{I}_{1}<\mathrm{IV}_{\mathrm{v}}$, while we have on the contrary I $>$ Iv for He. This is in agreement with our experlences showing that the plasma is always dominated by the gas in the case of $\mathrm{Ar}$ and $\mathrm{N2}$.

In He, at moderate intensity, breakdown only occurs in the metal vapour (erosion plasma) while there is no plasma developement in the gas. At higher laser Intensity, breakdown takes place both in metal vapour and gas.

Electron microscopy Investigations show that the process always involves a 1iquid phase. In He the surface shows a crater or a smooth surface depending on the pressure; in Ar a crystalline surface reorganization or surface damages are observed; in $\mathrm{N}_{2}$, a yellow compound is formed indicating the formation of TiN. Our future research will concern the compound layer symthesis formed with the aid of the laser plasma.
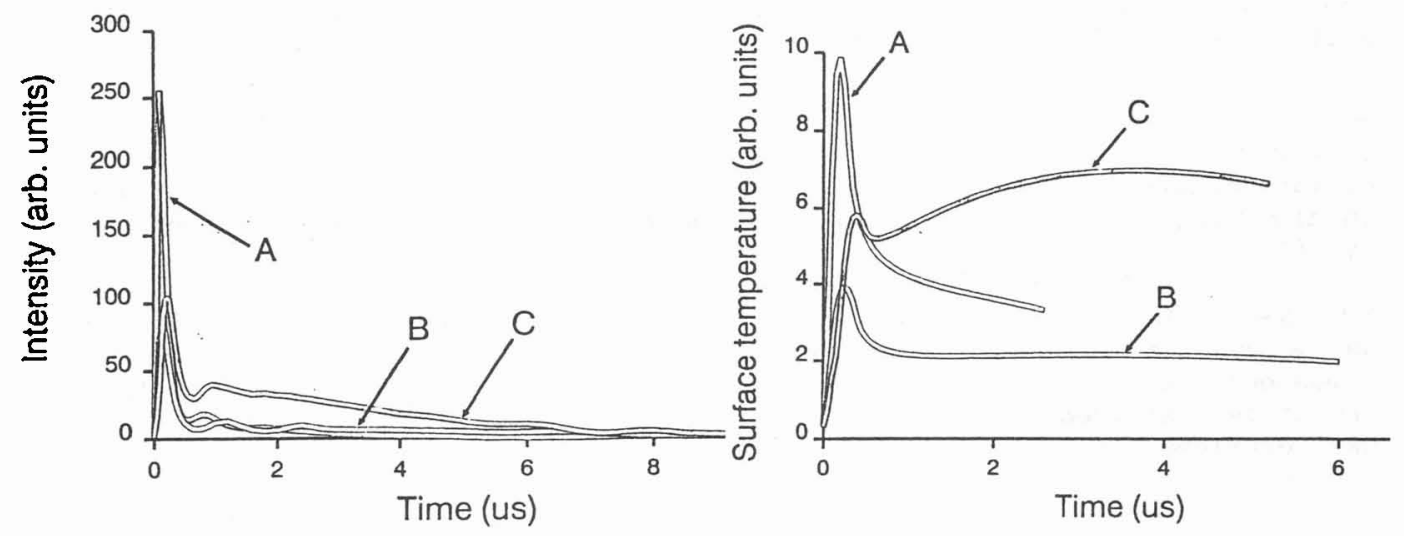

Figure 1: Laser pulse shapes

Figure 2: Surface temperature variation 